\title{
Intramuscular Ganglion of the Quadriceps Femoris
}

\author{
Yeung Jin Kim, $\mathrm{MD}^{1}$, Soo Uk Chae, $\mathrm{MD}^{1}$, Byong San Choi, $\mathrm{MD}^{1}$, Jong Yun Kim, $\mathrm{MD}^{1}$, and Hyang Jeong Jo, $\mathrm{MD}^{2}$ \\ Departments of ${ }^{1}$ Orthopaedic Surgery and ${ }^{2}$ Pathology, Gunsan Medical Center of Wonkwang University Hospital, Wonkwang University School of Medicine, Gunsan,
} Korea

Ganglion cysts are common lesions that are most often found around the joints of the hands and feet. Ganglia around the distal femur usually occur within the synovial membrane or tendon sheath, but rarely within muscles. Several cases of intramuscular ganglions in the hand and wrist have been reported, but a ganglion cyst in the quadriceps muscle has rarely been addressed in studies. In this report, we present a 17-year-old patient with a painful movable mass in the intramuscular area of the quadriceps femoris that was diagnosed by ultrasound and treated by excision and biopsy.

Keywords: Intramuscular ganglion cyst, Quadriceps muscle

Ganglion cysts are benign soft-tissue masses that usually originate from the joint capsule or tendon sheath and contain gellike fluid. The pathogenesis of ganglia is obscure. A ganglion cyst's connection with the joint capsule is formed due to secondary degeneration of the capsule. Brooks ${ }^{1)}$ suggested that a ganglion cyst may arise from extraarticular synovial remnants in the adjacent joints at the time of joint formation. In the orthopedic practice, soft tissue ganglia are commonly encountered in the dorsal wrist and lateral aspect of the hand, whereas intramuscular ganglia have rarely been reported ${ }^{1,2}$.

In this report, we present a case of intramuscular ganglion cyst in the quadriceps femoris that was treated with excision and biopsy with a review of the literature.

Received May 19, 2012; Revised (1st) November 5, 2012;

(2nd) November 26, 2012; Accepted January 8, 2013

Correspondence to: Byong San Choi, MD

Department of Orthopaedic Surgery, Gunsan Medical Center of

Wonkwang University Hospital, 27 Uiryowon-ro, Gunsan 573-713,

Korea

Tel: +82-63-472-5100, Fax: +82-63-472-5104

E-mail: yjkim1@wonkwang.ac.kr

This is an Open Access article distributed under the terms of the Creative Commons Attribution Non-Commercial License (http://creativecommons.org/licenses/by-nc/3.0/) which permits unrestricted non-commercial use, distribution, and reproduction in any medium, provided the original work is properly cited.

\section{Case Report}

A 17-year-old male patient visited our institution with a complaint of a painful movable mass on the lateral distal aspect of the right femur that was not caused by trauma. The mass had been slowly growing for 6 months and started to cause increasing pain from one month ago. The patient had no history of other systemic disease. In the physical examination, a firm and movable mass with a size of a quail egg was palpated in the lateral distal aspect of the right femur. The size of the mass did

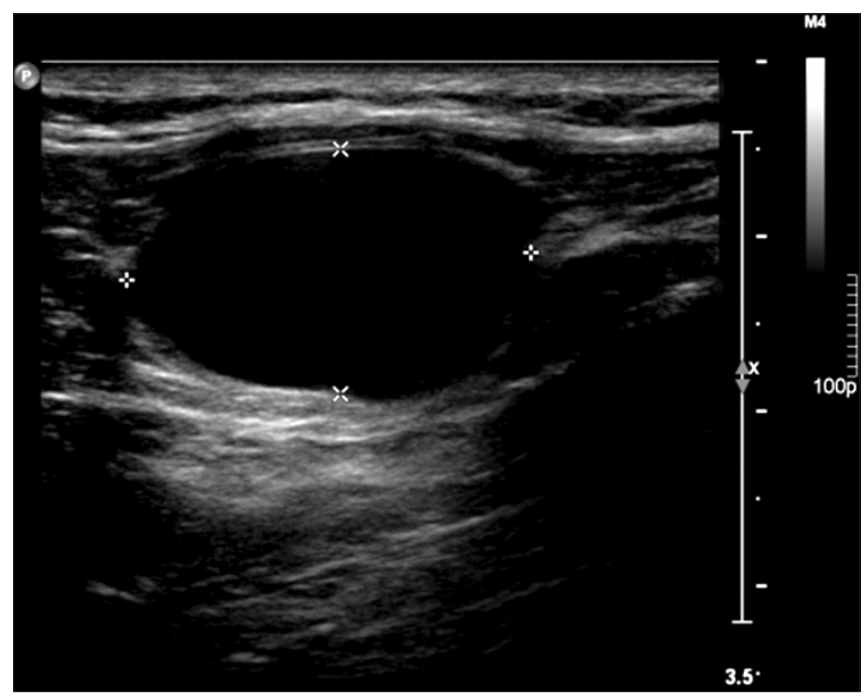

Fig. 1. Sonographic image showing the non-lobulated mass of a cystic lesion $(4.1 \times 2.5 \mathrm{~cm})$ within the vastus lateralis of the quadriceps femoris muscle. 


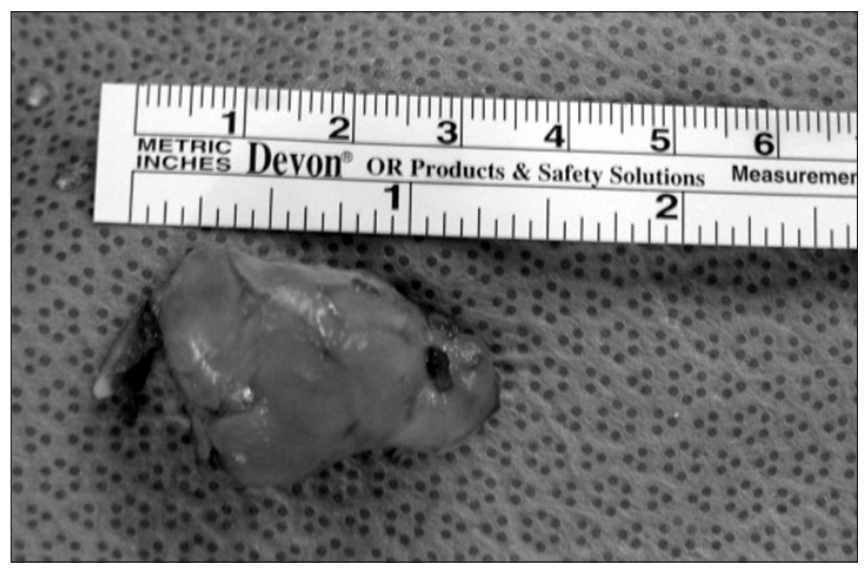

Fig. 2. The intramuscular ganglion in the vastus lateralis of the quadriceps femoris was excised.

not change during joint motion. Neither muscle weakness and numbness nor abnormal sensation was observed in the lower leg. In the absence of abnormal findings on radiography, ultrasound was performed, in which a cystic tumor approximately $4.1 \times 2.5$ $\mathrm{cm}$ in size was identified in the quadriceps femoris (Fig. 1). A magnetic resonance imaging (MRI) was considered unnecessary because it had a relatively distinct border and was well-encapsulated. Exploration and excision of the mass were performed under the assumption of a vascular lesion or a lipoma within the vastus lateralis of the quadriceps femoris. Noninvasive methods such as aspiration and steroid injection could be an option, but surgical excision was our treatment of choice in order to reduce the possibility of recurrence. Intraoperatively, spindleshaped swelling was observed and a firm mass was palpated in the vastus lateralis of the quadriceps femoris. The mass could be dissected with ease from the surrounding tissue and had a stalk as other ganglion cysts. When the muscle sheath was removed, the mass was found to originate from the muscle, not from the muscle sheath. In the naked eye, the intramuscular cystic mass was $3.4 \times 2.2 \times 1.4 \mathrm{~cm}$ in size, weighed $4.7 \mathrm{~g}$, and had some muscle tissue on its smooth surface (Fig. 2). When dissected upon excision, clear jelly-like fluid was noted in the sac. The biopsy results showed that the cystic wall was composed of fibrous tissue, lacked a synovial lining, and had muscular tissue on the surface, which led us to the diagnosis of an intramuscular ganglion cyst (Fig. 3). Rehabilitation was started after two weeks of splint immobilization. There has been neither recurrence nor complications for the 1 year and 6 months of follow-up period.

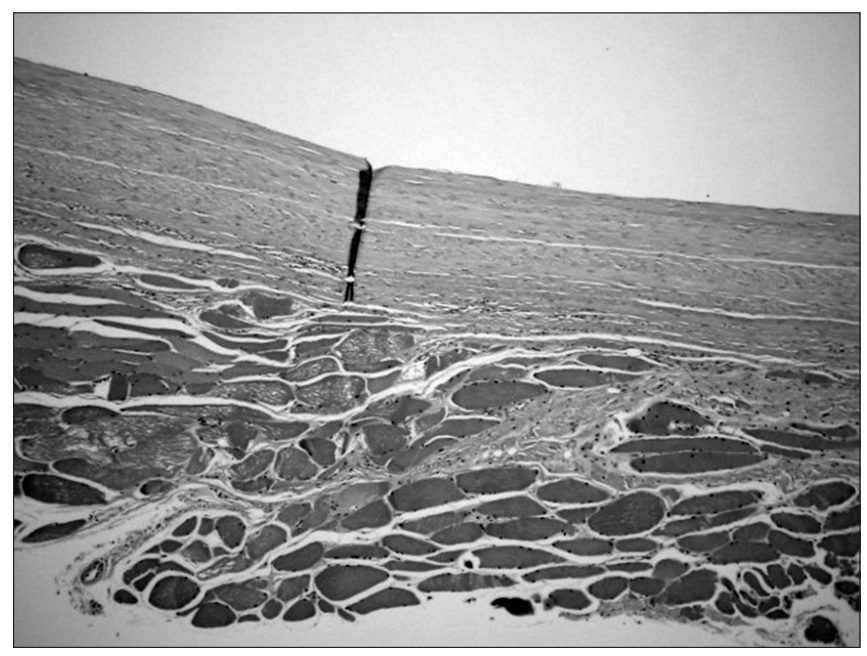

Fig. 3. A thick walled fibrous cyst with hypertrophic synovial cells was seen in histological study. The wall was composed of dense fibrous tissue, and lacked a synovial lining $(\mathrm{H} \& \mathrm{E}, \times 40)$.

\section{Discussion}

Ganglion cysts are the most common soft tissue lesions of the hand that are composed of a fluid-filled sac connected to a synovial sheath, a tendon sheath, or a tendon. The male to female prevalence ratio of ganglion cysts is $1: 3$. They can occur at any age especially in people in their teens and 30s. The pathogenesis of ganglion cysts has not yet been determined. Some studies associate them with mucoid degeneration of soft tissues, outpouchings of tissues through defects in the joint capsule or tendon sheath, or a trauma ${ }^{3}$. The most common area of occurrence is the radial side of the extensor digiti communis tendon on the dorsum, followed by the area between the flexor carpi radialis and the abductor pollicis longus tendons on the volar side of the wrist ${ }^{4}$. Ganglions cysts arise from joints in most cases and rarely from the tendon sheath, cartilage, cruciate ligaments, nerves, and muscles ${ }^{5-77}$. They have no communication with adjacent joint capsules in $30 \%$ of the cases ${ }^{5,788}$.

Intraarticular ganglion cysts in the knee have been rarely reported. Although most of the ganglia around the knee joint are located in the tendon sheath or joint capsule, they can form in the meniscus, anterior cruciate ligament, posterior cruciate ligament, and common peroneal nerve ${ }^{9)}$. Intraosseous ganglion cysts that are characteristically located in the epiphysis of long bones are mostly solitary and involve the distal end of the tibia. Currently, there are only 2 reported cases of ganglion cysts in the quadriceps femoris that were diagnosed using plain radiography, ultrasound, and computed tomography. In our patient, the diagnosis was relatively easily made using ultrasound only. 
The majority of ganglion cysts are palpable tender lumps without any accompanying symptoms. However, when a ganglion cyst exerts pressure on the nerves or blood vessels, pain, numbness, and muscle weakness may occur or tenderness and discomfort during joint motion may present from the initial stage.

Plain radiography is not reliable for the diagnosis of ganglion cysts. Although ultrasound is a valuable diagnostic tool, it is limited in its ability to evaluate the relationship between a cyst and adjacent soft tissues. MRI is essential for the differential diagnosis from other masses such as vascular lesions and lipomas. In our patient, MRI was considered unnecessary because the mass was seen well-encapsulated with a distinct border on ultrasound.

Ganglion cysts can regress spontaneously and recur occasionally. It is important to identify during surgery whether a lesion is a mass, has a connection with a joint, or is associated with intraarticular disease and to perform thorough excision in order to reduce the likelihood of recurrence. Ganglia rarely recur with proper curative measures, but inappropriate treatment methods can result in a recurrence rate of $50 \%{ }^{3)}$. The available treatment options range from non-surgical methods such as rupture by direct pressure, aspiration with or without a steroid injection, and draining using threads to surgical excision with or without arthroscopy.

The cure rate of ganglion cysts has no association with gender, age, and size and location. Nelson et al. ${ }^{4)}$ reported that the cure rate was $94 \%$ after excision under general anesthesia or axillary nerve anesthesia, $84 \%$ after excision under local anesthesia with a tourniquet in place, and $65 \%$ after rupture by direct pressure or aspiration with a steroid injection. In the study by Aydin et al. ${ }^{10)}$ on 40 cases of excision of ganglion cysts of the hand, the recurrence rate was $22 \%$, damage to the median palmar cutaneous nerve was observed in $10 \%$, radial nerve damage was present in 5\%, and joint stiffness occurred in 5\%. They cautioned that the possibility of recurrence and other complications should be taken into consideration in determining a surgical option for the treatment of a ganglion cyst.

Intramuscular ganglion cysts are rare with only a few cases involving the hand present in the literature. Ganglion cysts in the distal femur have been rarely reported. In this case report, we described a case of an intramuscular ganglion cyst in the vastus lateralis of the quadriceps femoris that was successfully treated with excision without complications.

\section{Conflict of Interest}

No potential conflict of interest relevant to this article was reported.

\section{Acknowledgments}

This work was supported by Wonkwang University 2012 Research Funds.

\section{References}

1. Brooks DM. Nerve compression by simple ganglia. J Bone Joint Surg Br. 1952;34:391-400.

2. Ahn JH, Suk SI, You MC. Intraneural ganglion of the peroneal nerve: a case report. J Korean Orthop Assoc. 1972;7: 344-6.

3. Angelides AC, Wallace PF. The dorsal ganglion of the wrist: its pathogenesis, gross and microscopic anatomy, and surgical treatment. J Hand Surg Am. 1976;1:228-35.

4. Nelson CL, Sawmiller S, Phalen GS. Ganglions of the wrist and hand. J Bone Joint Surg Am. 1972;54:1459-64.

5. Kang SY, Lee HJ, Lee SH. Intramuscular ganglion of the peroneus muscle mimicking peroneal compartment syndrome: a case report. J Korean Orthop Assoc. 2004;39:228-31.

6. Lee YU, Kook SH, Chung EC, Youn EK, Park JY. MRI of ganglion cysts in uncommon sites or with atypical appearance. J Korean Radiol Soc. 1999;41:393-9.

7. Yang SW, Teng HP, Tarng YW, Wong CY. Intramuscular ganglion cyst of the quadriceps muscle: report of a case. Mid Taiwan J Med. 2002;7:193-7.

8. Park S, Jin W, Chun YS, Park SY, Kim HC, Kim GY, Park JS, Ryu KN. Ruptured intramuscular ganglion cyst in the gastrocnemius medialis muscle: sonographic appearance. J Clin Ultrasound. 2009;37:478-81.

9. Muckle DS, Monahan P. Intra-articular ganglion of the knee: report of two cases. J Bone Joint Surg Br. 1972;54:520-1.

10. Aydin A, Kabakas F, Erer M, Ozkan T, Tuncer S. Surgical treatment of volar wrist ganglia. Acta Orthop Traumatol Turc. 2003;37:309-12. 